\title{
ORTHOSYMMETRIC ORTHOLATTICES
}

\author{
R. MAYET
}

(Communicated by Louis J. Ratliff, Jr.)

\begin{abstract}
Orthosymmetric ortholattices, which form an equational class of algebras, approximate ortholattices of closed subspaces of Hilbert spaces, and more generally projection lattices of von Neumann algebras, more closely than orthomodular lattices
\end{abstract}

The main interest in orthomodular lattices (abbreviated OMLs) comes from the fact that they constitute a simple algebraic approximation of closed subspace ortholattices $C(H)$ of Hilbert spaces and more generally of projection ortholattices of von Neumann algebras, and that they lead to logico-algebraic approaches to quantum mechanics $[2,7,8]$.

On a lattice $C(H)$ (where $H$ is a Hilbert space) there is, besides ortholattice operations $\wedge, \vee, \perp$, another simple binary operation $S:(X, Y) \rightarrow \sigma_{X}(Y)$, where $\sigma_{X}$ denotes the orthogonal symmetry operator corresponding to $X$ (defined, for $x=x_{1}+x_{2}, x_{1} \in X, x_{2} \in X^{\perp}$, by $\left.\sigma_{X}(x)=x_{1}-x_{2}\right)$.

In this paper we define a new variety obtained by adding to the theory of OMLs a new binary operation $S$ and new identity axioms. Algebras defined in this way are called orthosymmetric ortholattices (abbreviated OSOLs). The orthomodular identity is an easy consequence of new axioms (Proposition 1), hence every OSOL is an OML.

Besides lattices $C(H)$, all projection lattices of von Neumann algebras (Corollary of Proposition 12), and all Boolean algebras carry a natural structure of OSOL. Boolean algebras are just trivial OSOLs in which $S(a, b)=b$ for any $a, b$; this is an obvious consequence of the fact that, in any OSOL, $a$ commutes with $b$ iff $S(a, b)=b$. All finite modular ortholattices, but only few of the known finite nonmodular OMLs (in particular few of the finite horizontal sums of Boolean algebras), can be equipped with a structure of OSOL.

Just as regards OMLs, every interval of an OSOL carries a natural structure of OSOL, and zero equivalence classes of congruences on OSOLs are $p$-ideals. This last result is the most difficult to prove and its proof needs most of the previously stated results.

In $\S 6$ we extend the coordinatization theory for OMLs by means of Baer *semigroups $[4,5]$, (also called Foulis semigroups $[1,9])$ to OSOLs. With this

Received by the editors June 18, 1990 and, in revised form, September 18, 1990.

1980 Mathematics Subject Classification (1985 Revision). Primary 06C15, 81B10; Secondary $20 \mathrm{M} 07$.

Key words and phrases. Quantum logic, orthomodular lattices, Baer *-semigroups. 
aim, we define the variety of symmetric Baer ${ }^{*}$-semigroups. In this section we study an identity satisfied in every symmetric Baer ${ }^{*}$-semigroup. We show that this identity holds in any Baer *-semigroup coordinatizing an OML, but not in all Baer ${ }^{*}$-semigroups. This proves that the class of all Baer ${ }^{*}$-semigroups is not the smallest equational class containing all *-semigroups coordinatizing OMLs.

In the last paragraph we describe a simple idealized physical experiment showing that some orthogonal symmetries of the phase space in quantum mechanics have a physical meaning.

The reader can find information about ortholattices and OMLs in [9]. For any elements $a, b$ of an ortholattice, we use the following notation:

$$
\begin{aligned}
& \varphi_{a}(b)=\left(b \vee a^{\perp}\right) \wedge a \quad\left(\varphi_{a} \text { is called the Sasaki projection on } a\right) ; \\
& a \rightarrow b=(a \wedge b) \vee a^{\perp} ; \\
& a \leftrightarrow b=(a \wedge b) \vee\left(a^{\perp} \wedge b^{\perp}\right) ; \\
& \operatorname{Com}(a, b)=(a \wedge b) \vee\left(a^{\perp} \wedge b\right) \vee\left(a \wedge b^{\perp}\right) \vee\left(a^{\perp} \wedge b^{\perp}\right) .
\end{aligned}
$$

\section{BASIC DEFINITIONS AND PROPERTIES}

Definition. An OSOL is an ortholattice equipped with a binary operation $S$, satisfying the following axioms, where $S(a, b)$ is denoted by $S_{a}(b)$ :

(S1) For every $a \in L, S_{a}$ is an involutive automorphism of $(L, S)$ :

(a) $S_{a}\left(x^{\perp}\right)=S_{a}(x)^{\perp}$;

(b) $S_{a}(x \wedge y)=S_{a}(x) \wedge S_{a}(y)$;

(c) $S_{a} \circ S_{a}=1_{L}$;

(d) $S_{a} \circ S_{b} \circ S_{a}=S_{S_{a}(b)}$;

(S2) $x \vee S_{a}(x)=x \vee \varphi_{a}(x)$;

(S3) if $a \perp b$ then $S_{a \vee b}=S_{a} \circ S_{b}$.

Proposition 1. Every $O S O L$ is an $O M L$.

Proof. Let $(L, S)$ be an OSOL. If $x \in L$, then by (S2), $x \vee S_{1}(x)=x \vee \varphi_{1}(x)=$ $x$, hence $S_{1}(x) \leq x$. It follows that $x=S_{1}\left(S_{1}(x)\right) \leq S_{1}(x)$, thus $S_{1}(x)=x$ and $S_{1}=1_{L}$. By (S3) for each $a$ in $L, S_{a} \circ S_{a^{\perp}}=S_{1}=1_{L}$, which by (S1) implies $S_{a}=S_{a^{\perp}}$. It follows that, for any $x \in L, x \vee S_{a}(x)=x \vee S_{a^{\perp}}(x)$, thus by (S2), $x \vee \varphi_{a}(x)=x \vee \varphi_{a^{\perp}}(x)$, and it is easy (considering the case $x \leq a$ ) to show that this identity is equivalent to orthomodularity.

Proposition 2. The class of all OSOLs is an equational class.

Proposition 3. Let $H$ be a Hilbert space (over $\mathbf{R}$ or $\mathbf{C}$ ) and let $L=C(H)$. For all $X, Y$ in $L$, let $S(X, Y)$ be the image of $Y$ under the orthogonal symmetry corresponding to $X$. Then $(L, S)$ is an $O S O L$.

Proof. Let $X \in L, p_{X}$ be the projection operator onto $X$, and $\sigma_{X}=2 p_{X}-1_{H}$ the (orthogonal) symmetry operator corresponding to $X$. Operation $S$ on $L$ is defined by $S(X, Y)=S_{X}(Y)=\sigma_{X}(Y)=\left\{\sigma_{X}(y) \mid y \in Y\right\}$. Then $S_{X}(Y) \in L$, and it is easy to see that:

- $S_{X}$ is an involutive automorphism of $(L, S)$;

- $Y+S_{X}(Y)=Y+p_{X}(Y)$, and $\varphi_{X}(Y)$ is the topological closure of $p_{X}(Y)$, hence $Y \vee S_{X}(Y)=Y \vee \varphi_{X}(Y)$;

- if $X \perp Y$ then $\sigma_{X} \circ \sigma_{Y}=-\sigma_{X \vee Y}$, hence $S_{X} \circ S_{Y}=S_{X \vee Y}$. 
Proposition 4. Proposition 3 can be extended to any orthomodular space over a division ring of characteristic different from 2 .

Proof. Let $H$ be a vector space over a division ring $K$ whose characteristic is $\neq 2$, with an involutive antiautomorphism, equipped with a definite Hermitian form. A subspace $X$ of $H$ is said to be closed if $X=X^{\perp \perp}$. Let $L=C(H)$ be the ortholattice of all closed subspaces of $H$. Then $H$ is said to be an orthomodular space [6], or a Hilbert space over $K$ [10], if $L$ is orthomodular or, equivalently, if for any $X \in L, H=X+X^{\perp}$.

If $H$ is an orthomodular space, then for each $X \in L$, the orthogonal projection $p_{X}$ is defined by $p_{X}(x)=x_{1}$, where $x=x_{1}+x_{2}, x_{1} \in X$ and $x_{2} \in X^{\perp}$. It is easy to see that $\varphi_{X}(Y)=p_{X}(Y)^{\perp \perp}$ for any $Y \in L$.

We define $X$ and $S_{X}$ as above and in the same way show that $(L, S)$ is an OSOL, the only difference being that the topological closure of $E \subset H$ is replaced by $E^{\perp \perp}$.

Corollary. Every complete, atomic, irreducible OML of dimension $\geq 4$, satisfying the covering law, can be equipped with a structure of $O S O L$.

Proof. Such an OML is isomorphic to an ortholattice $C(H)$ whose $H$ is an orthomodular space [10].

In the following, $(L, S)$ denotes an OSOL.

Proposition 5. For all $a, b$ in $L$,

(1) $S_{a}=S_{a^{\perp}}$.

(2) The following statements are equivalent:

(a) $a C b$;

(b) $S_{a}(b)=b$;

(c) $S_{a} \circ S_{b}=S_{a \leftrightarrow b}$;

(d) $S_{a} \circ \varphi_{b}=\varphi_{b} \circ S_{a}$.

(3) $a \in C(L)$ if and only if $S_{a}=1_{L}$.

Proof. (1) has been proved in Proposition 1.

(2) (a) $\Leftrightarrow$ (b). If $a C b$ then $b=b \vee \varphi_{a}(b)=b \vee S_{a}(b)$, thus $S_{a}(b) \leq b$, and therefore, $S_{a}(b)=b$. Conversely, if $S_{a}(b)=b$ then $b=b \vee S_{a}(b)=$ $b \vee \varphi_{a}(b)=(b \vee a) \wedge\left(b \vee a^{\perp}\right)$, hence $a C b$.

(a) $\Rightarrow$ (c). If $a C b$ then $a=(a \wedge b) \vee\left(a \wedge b^{\perp}\right), b^{\perp}=\left(a \wedge b^{\perp}\right) \vee\left(a^{\perp} \wedge b^{\perp}\right)$, hence by axioms (S1), (S3) and above results $S_{a} \circ S_{b}=S_{a} \circ S_{b^{\perp}}=S_{a \wedge b^{\circ}} \circ S_{a \wedge b^{\perp}} \circ$ $S_{a \wedge b^{\perp}} \circ S_{a^{\perp} \wedge b^{\perp}}=S_{a \wedge b} \circ S_{a^{\perp} \wedge b^{\perp}}=S_{(a \wedge b) \vee\left(a^{\perp} \wedge b^{\perp}\right)}=S_{a \leftrightarrow b}$.

(c) $\Rightarrow$ (b). If $S_{a} \circ S_{b}=S_{a \mapsto b}$, then as $b \quad C \quad a \leftrightarrow b, S_{a}(b)=S_{a}\left(S_{b}(b)\right)=$ $S_{a \leftrightarrow b}(b)=b$.

(b) $\Leftrightarrow$ (d). It is easy to see that for any $a, b$ in $L, S_{a} \circ \varphi_{b} \circ S_{a}=\varphi_{S_{a}(b)}$. Therefore, if $S_{a}(b)=b, S_{a} \circ \varphi_{b} \circ S_{a}=\varphi_{b}$, thus $S_{a} \circ \varphi_{b}=\varphi_{b} \circ S_{a}$. Conversely, if $S_{a} \circ \varphi_{b}=\varphi_{b} \circ S_{a}$, then $S_{a} \circ \varphi_{b} \circ S_{a}=\varphi_{b}=\varphi_{S_{a}(b)}$, hence $S_{a}(b)=b$.

(3) is an obvious consequence of the above equivalence (a) $\Leftrightarrow(\mathrm{b})$.

\section{EXAMPLES}

(a) On a Boolean lattice $L_{1}$, there exists a unique structure of OSOL, obtained by defining $S_{a}$ for every $a$ in $L_{1}$ by $S_{a}=1_{L_{1}}$. 
(b) Let $n \geq 2$ and let $D$ be a one-dimensional subspace of $\mathbf{R}^{2}$. The subOML of $C\left(\mathbf{R}^{2}\right)$ whose atoms are all one-dimensional subspaces obtained from $D$ by $k \pi / 2 n$ rotations $(0 \leq k<2 n)$ is a sub-OSOL of $C\left(\mathbf{R}^{2}\right)$, which as an OML is isomorphic to $M O_{n}$.

This shows that for each $n \geq 2, M O_{n}$ can be equipped with a structure of OSOL. Actually for $n \geq 3$, there are more than one structure of OSOL on $M O_{n}$. Indeed for any cardinal $\alpha \geq 3$, if we define $S_{a}$ for any atom $a$ of $M O_{\alpha}$ by $S_{a}(b)=b^{\perp}$ if $b$ is an atom such that $b \neq a, a^{\perp}, S_{a}(b)=b$ otherwise, then it is not difficult to verify that $\left(M O_{\alpha}, S\right)$ is an OSOL, and that if $\alpha$ is an integer, this OSOL is not isomorphic to the previous one.

(c) Suppose an OSOL $L$ is the horizontal sum of a family $\left(B_{i}\right)_{i \in I}$ of Boolean algebras, each having at least 4 elements, where $\operatorname{Card}(I) \geq 2$. Let $i, j \in I$, $i \neq j$, and let $a \in B_{i}-\{0,1\}$. If $S_{a}\left(B_{j}\right)=B_{j}$, then for each $b \in B_{j}-\{0\}$, $b \vee S_{a}(b)=b \vee \varphi_{a}(b)=b \vee a=1$, hence $S_{a}(b) \geq b^{\perp}$; it follows that if $c$, $d \in B_{j}$ are such that $c \neq 0, d \neq 0, c \wedge d=0$, then $S_{a}(c \vee d)=S_{a}(c) \vee S_{a}(d) \geq$ $c^{\perp} \vee d^{\perp}=1$, thus $c \vee d=1$, which proves that $B_{j}$ is four-element.

Let us assume that there exists $p \in I$ such that $\operatorname{Card}\left(B_{p}\right)>4$. By the above result, there exist $q \neq p$ and $e \in L$ such that $S_{e}\left(B_{p}\right)=B_{q}$, and so $B_{q}$ is isomorphic to $B_{p}$. Let $a, b \in B_{p}-\{0,1\}$ such that $a \neq b, a \neq b^{\perp}$. By (2) $a C b$ implies $S_{a} \circ S_{b}=S_{a \leftrightarrow b}$. Moreover, from $a \neq b, b^{\perp}$ it follows that $a \leftrightarrow b \in B_{p}-\{0,1\}$. If we should have $S_{a}\left(B_{q}\right)=S_{b}\left(B_{q}\right)$, it would follow that $S_{a \leftrightarrow b}\left(B_{q}\right)=B_{q}$, which is impossible by the above result and the assumption $\operatorname{Card}\left(B_{q}\right)>4$. Hence $S_{a}\left(B_{q}\right) \neq S_{b}\left(B_{q}\right)$.

Let $J$ be the set of all $j \in I$ such that $B_{j}$ is isomorphic to $B_{p}$. By the previous result, the map $\theta$ from $B_{p}$ to $J-\{p\}$ defined by $S_{a}\left(B_{q}\right)=B_{\theta(a)}$ is such that:

$(\theta(a)=\theta(b)) \Leftrightarrow\left(a=b\right.$ or $\left.a=b^{\perp}\right)$ (this is trivially true if $a \in\{0,1\}$ or $b \in$ $\{0,1\})$. It follows that $\operatorname{Card}\left(B_{p}\right)+2 \leq 2 \operatorname{Card}(J)$. Hence if $B_{p}$ is infinite, then $I$ is infinite, $\operatorname{Card}(I) \geq \operatorname{Card}\left(B_{p}\right)$, and therefore $\operatorname{Card}(I) \geq \operatorname{Sup}_{k \in I} \operatorname{Card}\left(B_{k}\right)$. In particular, if $I$ is finite, then for each $i \in I, B_{i}$ is finite.

From now on we suppose that $I$ is finite and consequently that each $B_{i}$ is finite. Let us assume that there exist $p, q \in I$ such that $B_{p}$ and $B_{q}$ are not isomorphic. We may assume, for instance, that $\operatorname{Card}\left(B_{q}\right)>4$. Let $P$ (resp. $Q$ ) be the set of all $i \in I$ such that $B_{i}$ is isomorphic to $B_{p}$ (resp. to $B_{q}$ ). If $a \in B_{p}-\{0,1\}$ then $S_{a}$ swaps each $B_{i}$, for $i \in Q$, with another. Hence $\operatorname{Card}(Q)$ is an even number. If $b \in B_{q}-\{0,1\}$ then $S_{b}$ swaps each $B_{i}$, for $i \in Q-\{q\}$ with another, thus $\operatorname{Card}(Q)$ is an odd number, which is a contradiction.

It follows that for all $i, j \in I, B_{i}$ is isomorphic to $B_{j}$. Moreover, if $\operatorname{Card}\left(B_{i}\right)>4$ then, by the above result, $\operatorname{Card}\left(B_{i}\right) \leq 2(\operatorname{Card}(I)-1)$. We also notice that, if $\operatorname{Card}\left(B_{i}\right)>4$, the above proof (in the case where $b \in B_{q}-\{0,1\}$ ) shows that $\operatorname{Card}(I)$ is an odd number.

Let us summarize these last results:

Proposition 6. Let $L$ be an $O S O L$ which is the horizontal sum of $n$ Boolean lattices $B_{1}, B_{2}, \ldots, B_{n}$. Then

(i) All Boolean lattices $B_{i}$ are finite and pairwise isomorphic,

(ii) If $\operatorname{Card}\left(B_{i}\right)>4$, then $n$ is an odd number and $n>\operatorname{Card}\left(B_{i}\right) / 2$. 
(d) It follows from Proposition 6 that the least OML fulfilling the following three requirements:

- $L$ is the horizontal sum of a finite number of Boolean lattices,

- $L$ is not isomorphic to any $M O_{n}$.

- $L$ can be equipped with a structure of OSOL, possesses at least 5 blocks, each of them with at lest 3 atoms.

Let $\operatorname{HOR}(5,3)$ (see Figure 1) be the horizontal sum of 5 Boolean algebras, each of them with 3 atoms.

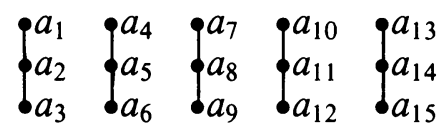

FIGURE 1

We describe any involutive automorphism $f$ of $\operatorname{HOR}(5,3)$ by giving the list of all pairs $(i, j), i \neq j$, such that atoms $a_{i}, a_{j}$ are swapped by $f$. A computer calculation shows that there is a unique OSOL structure $(\operatorname{HOR}(5,3), S)$ such that:

$$
\begin{aligned}
& S_{a_{1}}=(4,7)(5,8)(6,9)(10,13)(11,14)(12,15), \\
& S_{a_{2}}=(4,13)(5,14)(6,15)(7,10)(8,11)(9,12), \\
& S_{a_{4}}=(1,7)(2,8)(3,9)(10,15)(11,13)(12,14) .
\end{aligned}
$$

It is easy to prove the uniqueness of this structure since, for instance, we have by axiom $(\mathrm{S} 1)(\mathrm{d})$,

$$
S_{a_{7}}=S_{S_{a_{1}}\left(a_{4}\right)}=S_{a_{1}} \circ S_{a_{4}} \circ S_{a_{1}}
$$

and in the same way one can express $S_{a_{i}}$ successively for $i=13,8,10,11,5$, $15,14,6,9,3$.

(e) If a finite OML is given by a Greechie diagram, it is easy in most cases to show that it cannot be equipped with a structure of OSOL. For instance, let us assume that $(L 7, S)$ where $L 7$ is the OML given in Figure 2, is an OSOL.

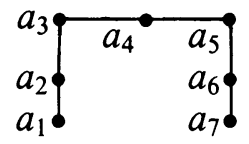

FIGURE 2

Then, as $a_{1}$ does not commute with $a_{4}$, by (2), we have $S_{a_{1}}\left(a_{4}\right) \neq a_{4}$, which is a contradiction since $a_{4}$ is invariant under each automorphism of $L 7$.

(f) OMLs $L 9$ and $L 15$ given in Figures 3 and 4 can be equipped with OSOL structures.

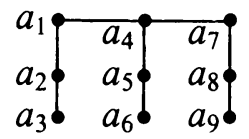

FIGURE 3

Operation $S$ on $L 9$ can be entirely defined, for instance, by:

$$
\begin{aligned}
& S_{a_{2}}=(4,7)(5,8)(6,9), \\
& S_{a_{5}}=(1,7)(2,8)(3,9) .
\end{aligned}
$$

Notation used here is the same as above in section (d), and this result may be proved in the same way, observing that necessarily $S_{a_{1}}=(5,6)(8,9)$. 


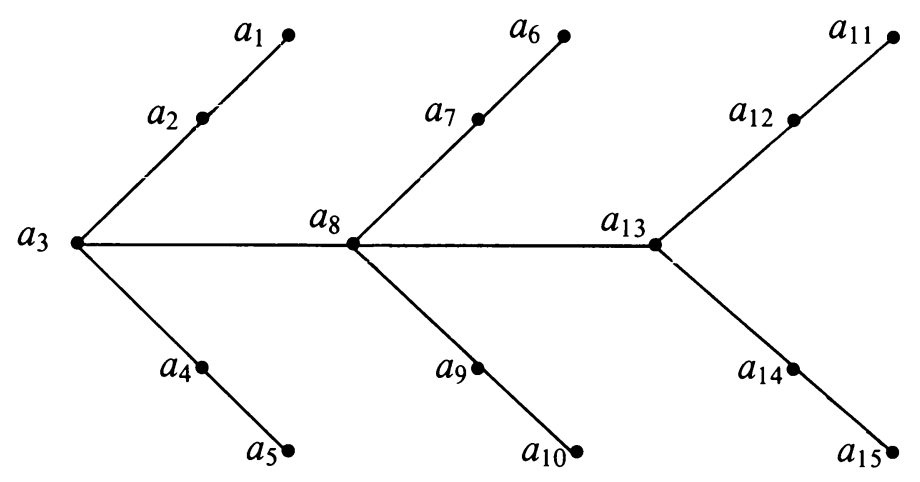

FIGURE 4

On OML $L 15$, operation $S$ is determined by conditions:

$$
\begin{aligned}
& S_{a_{1}}=(4,5)(6,11)(7,12)(8,13)(9,14)(10,15), \\
& S_{a_{3}}=(6,7)(9,10)(11,12)(14,15), \\
& S_{a_{5}}=(1,2)(6,14)(7,15)(8,13)(9,12)(10,11), \\
& S_{a_{6}}=(9,10)(1,11)(2,12)(3,13)(4,15)(5,14) .
\end{aligned}
$$

It is easy to see that, in both cases, the OSOL structure is not unique.

(g) As OSOLs form a variety, we can obtain other examples of finite nonmodular OSOLs by constructing products of above examples, for instance the product of $\operatorname{HOR}(5,3)$ with the two-element Boolean algebra.

\section{INDEPENDENCE OF AXIOMS}

Proposition 7. Each of axioms $(S 1)(d),(S 2),(S 3)$ is independent of all other axioms of OSOLs.

Proof. Following results (a) and (c) have been obtained by computer.

(a) on OML $L 15$ given in Figure 4, we define a binary operation $S^{\prime}$ in the same way as $S$ above, except that $S_{a_{1}}^{\prime}=S_{a_{1}^{\perp}}^{\prime}=f \circ S_{a_{1}} \circ f$ and $S_{a_{2}}^{\prime}=S_{a_{2}^{\perp}}^{\prime}=$ $f \circ S_{a_{2}} \circ f$ where $f$ is the involutive automorphism of $L 15$, which swaps $a_{11}$ and $a_{12}$, all other atoms being invariant under $f$. Then $\left(L 15, S^{\prime}\right)$ fulfills all OSOL axioms, except $(\mathrm{S} 1)(\mathrm{d})$.

(b) Let $L$ be a non-Boolean OML. If we define $S$ by $S_{a}=1_{L}$ for every $a$ in $L$, then $(S, L)$ satisfies all axioms of OSOLs but (S2). This shows the significance of axiom (S2) without which every OML would carry a trivial structure of OSOL.

(c) On OML L9 given in Figure 3, we define the binary operation $S^{\prime}$ as follows:

- $S_{0}^{\prime}=S_{1}^{\prime}=1_{L 9}$

- for each atom $a, S_{a}^{\prime}=S_{a}$, where $S$ is defined as above, and $S_{a \perp}^{\prime}=$ $S_{\theta(a)}$, where $\theta$ is the involutive automorphism of $\mathrm{L} 9$, which swaps each of the couples $\left(a_{1}, a_{2}\right),\left(a_{4}, a_{5}\right),\left(a_{7}, a_{8}\right)$.

Then all OSOL axioms are satisfied in $\left(L 9, S^{\prime}\right)$ except (S3), since for instance, $S_{a_{1}}^{\prime} \circ S_{a_{1}^{\perp}}^{\prime} \neq S_{1}^{\prime}$. 


\section{SOME OTHER PROPERTIES OF OSOLS}

Proposition 8. Axioms ( $S 2)$ is equivalent to each of the following:

$\left(S^{\prime} 2\right) \quad b \wedge S_{a}(b)=b \wedge(a \rightarrow b)$;

$\left(S^{\prime \prime} 2\right) \varphi_{b^{\perp}} \circ S_{a} \circ \varphi_{b}=\varphi_{b^{\perp}} \circ \varphi_{a} \circ \varphi_{b}$.

Proof. Axiom (S2) is equivalent to $\left(b^{\perp} \vee S_{a}\left(b^{\perp}\right)\right)^{\perp}=\left(b^{\perp} \vee \varphi_{a}\left(b^{\perp}\right)\right)^{\perp}$, thus to $b \wedge S_{a}(b)=b \wedge(a \rightarrow b)$.

Let $L$ be an OSOL, and $a, b, x \in L$. Let $y=\varphi_{b}(x)$. Then $\varphi_{b^{\perp}}(y)=0$ hence:

$$
\varphi_{b^{\perp}}\left(S_{a}(y)\right)=\varphi_{b^{\perp}}\left(y \vee S_{a}(y)\right)=\varphi_{b^{\perp}}\left(y \vee \varphi_{a}(y)\right)=\varphi_{b^{\perp}}\left(\varphi_{a}(y)\right),
$$

which proves that $\left(S^{\prime \prime} 2\right)$ holds. Conversely, let $L$ be an OML equipped with a binary operation $S$ fulfilling condition $\left(S^{\prime \prime} 2\right)$. Then for any $a, b \in L$,

$$
\varphi_{b^{\perp}}\left(S_{a}(b)\right)=\varphi_{b^{\perp}}\left(S_{a}\left(\varphi_{b}(b)\right)\right)=\varphi_{b^{\perp}}\left(\varphi_{a}(b)\right),
$$

which can be written $\left(S_{a}(b) \vee b\right) \wedge b^{\perp}=\left(\varphi_{a}(b) \vee b\right) \wedge b^{\perp}$. It follows that

$$
S_{a}(b) \vee b=\left(\left(S_{a}(b) \vee b\right) \wedge b^{\perp}\right) \vee b=\left(\left(\varphi_{a}(b) \vee b\right) \wedge b^{\perp}\right) \vee b=\varphi_{a}(b) \vee b .
$$

Proposition 9. For any $a, b, x$ in $L$,

(4) $S_{a}=S_{b}$ iff $a C b$ and $a \leftrightarrow b \in C(L)$;

(5) if $L$ is irreducible then $S_{a}=S_{b}$ iff $a=b$ or $a=b^{\perp}$,

(6) $b \leftrightarrow S_{a}(b)=\operatorname{Com}(a, b)$;

(7) $S_{a} \circ S_{b}=S_{b} \circ S_{a}$ iff $\left(b C S_{a}(b)\right.$ and $\left.\operatorname{Com}(a, b) \in C(L)\right)$;

(8) $b C S_{a}(b)$ iff $S_{a}(b)=T_{a}(b)$, where $T_{a}(b)=\left(a^{\perp} \wedge b\right) \vee(a \wedge b) \vee\left(\left(a^{\perp} \vee b\right)\right.$ $\left.\wedge(a \vee b) \wedge b^{\perp}\right)$

(9) $b \perp S_{a}(b)$ iff $S_{a}(b)=\left(a^{\perp} \vee b\right) \wedge(a \vee b) \wedge b^{\perp}$;

(10) $a \wedge b=a \wedge S_{a}(b), a \vee b=a \vee S_{a}(b), a \rightarrow b=a \rightarrow S_{a}(b), \operatorname{Com}(a, b)=$ $\operatorname{Com}\left(a, S_{a}(b)\right)$;

(11) $S_{a}(x)=S_{\varphi_{a}(x)}(x)$.

Remarks. (a) By (4), the relation ( $a C b$ and $a \leftrightarrow b \in C(L))$ is an equivalence. It can be shown that on any OML, this relation is an equivalence.

(b) We have shown in $\S 2(\mathrm{~b})$ that for any cardinal $\alpha, \mathrm{MO}_{\alpha}$ can be equipped with a structure of OSOL $\left(\mathrm{MO}_{\alpha}, S\right)$, such that for all $a, b$ in $\mathrm{MO}_{\alpha}, S_{a}(b)=b$ or $S_{a}(b)=b^{\perp}$, hence in particular $S_{a}(b) C b$. It follows from (8) that in this case $S=T$, and more generally, that if $L$ is any OML belonging to the variety $V$ generated by all $\mathrm{MO}_{\alpha}$ (in particular if $L$ is any finite modular ortholattice), then $(L, T)$ is an OSOL. If $L$ is any OML, $(L, T)$ is not necessarily an OSOL. However if $a, b \in L$, then the subalgebra of $L$ generated by $\{a, b\}$ is in $V$, and it follows that every OSOL equation in two variables is satisfied in $(L, T)$.

Proposition 10. (12) $x \leq \operatorname{Com}(a, b)$ implies $S_{a} \circ S_{b}(x)=S_{a \leftrightarrow b}(x)$.

Proof. We first notice that in any OML, $x \leq y$ implies $\varphi_{b}(x)=\varphi_{\varphi_{b}(y)}(x)$. If $x \leq \operatorname{Com}(a, b)$, then by $(11)$, this identity implies $S_{b}(x)=S_{\varphi_{b}(\operatorname{Com}(a, b))}(x)$. As $\varphi_{b}(\operatorname{Com}(a, b))=b \wedge \operatorname{Com}(a, b)=(a \wedge b) \vee\left(a^{\perp} \wedge b\right)$, it follows that:

$$
S_{b}(x)=S_{(a \wedge b) \vee\left(a^{\perp} \wedge b\right)}(x)=S_{a \wedge b} \circ S_{a^{\perp} \wedge b}(x)=S_{a^{\perp} \wedge b} \circ S_{a \wedge b}(x) .
$$


As $S_{b}(x) \leq \operatorname{Com}(a, b)$, we can apply this last result to $b, a, S_{b}(x)$ instead of $a, b, x$, and we obtain:

$$
\begin{aligned}
S_{a}\left(S_{b}(x)\right) & =S_{a \wedge b^{\perp}} \circ S_{a \wedge b}\left(S_{b}(x)\right)=S_{a \wedge b^{\perp}} \circ S_{a \wedge b} \circ S_{a \wedge b} \circ S_{a^{\perp} \wedge b}(x) \\
& =S_{a \wedge b^{\perp}} \circ S_{a^{\perp} \wedge b}(x)=S_{a^{\perp} \leftrightarrow b}(x) .
\end{aligned}
$$

But $S_{a}=S_{a^{\perp}}$ and $\operatorname{Com}(a, b)=\operatorname{Com}\left(a, b^{\perp}\right)$, and it follows:

$$
S_{a} \circ S_{b}(x)=S_{a^{\perp}} \circ S_{b}(x)=S_{a \leftrightarrow b}(x) .
$$

\section{INTERVALS, CONGRUENCES, AND SUBALGEBRAS}

Proposition 11. Every interval of an OSOL possesses a natural OSOL structure. Proof. If $(L, S)$ is an OSOL, then on each interval $[a, b]$ of $L$, there is a natural OML structure. For $x, y \in[a, b], a=S_{x}(a) \leq S_{x}(y) \leq S_{x}(b)=b$. If for each $x$ in $[a, b]$ we denote by $S_{x}^{\prime}$ the restriction of $S_{x}$ to $[a, b]$, then it is easy to see that $\left([a, b], S^{\prime}\right)$ is an OSOL.

Proposition 12. Let $G$ be an ideal (resp. a filter) of $L$. Then $G$ is a p-ideal (resp. a p-filter) if and only if $G$ is stable under every automorphism $S_{a}$ for $a \in L$.

Proof. An ideal $G$ of $L$ is a $p$-ideal iff for all $x \in G$ and $a \in L, \varphi_{a}(x) \in G$. Hence we need only use axiom (S2).

Proposition 13. In an OSOL, zero equivalence classes of congruences are $p$ ideals.

Proof. It is obvious that such an equivalence class is a $p$-ideal. Conversely, let $I$ be a $p$-ideal of an OSOL $(L, S)$, let $F=\left\{x^{\perp} \mid x \in I\right\}$ the corresponding $p$-filter, and $\equiv$ be the OML congruence defined by: $x \equiv y \Leftrightarrow(x \leftrightarrow y \in F)$. We need only show that $\equiv$ is compatible with $S$.

If $x, y, a \in L$, and $x \equiv y$, then $S_{a}(x \leftrightarrow y)=S_{a}(x) \leftrightarrow S_{a}(y)$, and it follows by Proposition 12, that $S_{a}(x) \equiv S_{a}(y)$.

If $z \in L$ and $c \in F$, then by (6), $S_{c}(z) \leftrightarrow z=\operatorname{Com}(c, z)$. But $c \in F$ implies $\operatorname{Com}(c, z) \in F$, and it follows that $S_{c}(z) \equiv z$.

Let $a, b \in L$ such that $a \equiv b$. Let $x \in L$, and $y=x \wedge(a \leftrightarrow b)$. Then $a \leftrightarrow b \equiv 1$ implies $x \wedge(a \leftrightarrow b) \equiv x$, thus $y \equiv x$. As $a \leftrightarrow b \leq \operatorname{Com}(a, b)$, we have $y \leq \operatorname{Com}(a, b)$, hence by (12), $S_{a \leftrightarrow b}(y)=S_{a} \circ S_{b}(y)$. It follows from the above results and from $a \leftrightarrow b \in F$ that $S_{a \leftrightarrow b}(y) \equiv y$, therefore $S_{a} \circ S_{b}(y) \equiv y$ and $S_{b}(y) \equiv S_{a}(y)$. From $x \equiv y$ it follows that $S_{a}(x) \equiv S_{a}(y)$ and $S_{b}(x) \equiv S_{b}(y)$. Hence $S_{a}(x) \equiv S_{b}(x)$, and the proof is complete.

Corollary. The class of all OMLs that can be equipped with an OSOL structure is axiomatizable in the first order language of OMLs.

Proof. This class $C$ is obviously closed under formation of products, and by Proposition 13, is also closed under homomorphic images. It follows that $C$ is closed under ultraproducts, because an ultraproduct of algebras is a homomorphic image of a product. Hence $C$ is axiomatizable [3].

Remarks. (a) The class $C$, being closed under homomorphic images, admits a set of axioms that are positive sentences [3] (a sentence is said to be positive if it can be written using only connectives "and," "or," $\forall, \exists$ ). 
(b) $C$ is not a variety, because $C$ is not closed under subalgebras. For instance, $L 7$ (cf. Figure 2) is a sub-OML of OSOL $L 9$, and $L 7$ does not belong to $C$.

Proposition 14. If $L$ is an $O S O L$ and $E$ a subset of $L$, then $C(E)=\{x \in$ $L \mid \forall y \in E, x C y\}$ is a (relatively complete) sub-OSOL of $L$.

Proof. If $a, x \in C(E)$, then for any $y \in E$,

$$
\begin{aligned}
\operatorname{Com}\left(S_{a}(x), y\right) & =\operatorname{Com}\left(S_{a}(x), S_{a}(y)\right) \\
& =S_{a}(\operatorname{Com}(x, y))=S_{a}(1)=1 .
\end{aligned}
$$

Hence $S_{a}(x) \in C(E)$.

Corollary. Projection lattices of von Neumann algebras are OSOLs.

Proof. If $L$ is the projection ortholattice of a von Neumann algebra on a Hilbert space $H$, then in $C(H), L=C(C(L))$.

\section{SYMMETRIC BAER ${ }^{*}$-SEMIGROUPS}

In this section, most of the proofs that are rather standard are omitted.

Lemma. Let $L_{1}$ be an $O M L$ and $\Sigma\left(L_{1}\right)$ be its coordinate Baer *-semigroup [4]. For any map $f$ from $L_{1}$ to $L_{1}$, the following are equivalent:

(i) $f$ is an involutive automorphism of $L_{1}$;

(ii) $f \in \Sigma\left(L_{1}\right), f^{*}=f$, and $f^{2}=1_{L_{1}}$.

In what follows, $(L, S)$ denotes an OSOL, and $\Sigma(L)$ the coordinate Baer *-semigroup of the OML $L$.

Definition. We define on $\Sigma(L)$ a unary operation $\sigma$ by $f^{\sigma}=S_{f^{+}(0)}$, where $f^{+}(0)$ denotes the greatest element of $\{x \in L \mid f(x)=0\}$. (Recall that $f^{\prime}=$ $\left.\varphi_{f^{+}(0)}\right)$.

Proposition 15. The following identities are satisfied in $(\Sigma(L), \sigma)$ :

(SB1) (a) $f^{\sigma}=f^{\prime \sigma}$;

(b) $\left(f^{\sigma}\right)^{2}=1_{L}$;

(c) $\left(f^{\sigma}\right)^{*}=f^{\sigma}$;

(d) $f^{\sigma} g^{\sigma} f^{\sigma}=\left(g f^{\sigma}\right)^{\sigma}$

(SB2) $g f^{\sigma} g^{\prime}=g f^{\prime} g^{\prime}$;

(SB3) $\left(f(g f)^{\prime}\right)^{\sigma}=f^{\sigma}(g f)^{\prime \sigma}$.

Remark. Identities (SB2) and (SB3) can also be written resp. $g^{\prime \prime} f^{\sigma} g^{\prime}=g^{\prime \prime} f^{\prime} g^{\prime}$ (since, in any Baer ${ }^{*}$-semigroup, $\left.g=g g^{\prime \prime}\right)$, and $\left(f(g f)^{\prime}\right)^{\sigma}=f^{\sigma}(g f)^{\sigma}$.

Definition. A symmetric Baer *-semigroup is a Baer *-semigroup equipped with a unary operation $\sigma$ fulfilling identities (SB1), (SB2), (SB3) of Proposition 15.

We remind that if $\Sigma$ is a Baer ${ }^{*}$-semigroup and if $P^{\prime}(\Sigma)$ denotes the OML of its closed projections, then the map $\Phi$ from $\Sigma$ to $\Sigma\left(P^{\prime}(\Sigma)\right)$, defined by $\Phi_{f}(x)=\left(x f^{*}\right)^{\prime \prime}$, is a homomorphism of Baer ${ }^{*}$-semigroups. Moreover, if $f \in P^{\prime}(\Sigma)$, then $\Phi_{f}=\varphi_{f}$ (Sasaki projection on $f$ ). 
Proposition 16. Let $(\Sigma, \sigma)$ be a symmetric Baer *-semigroup. We define $S_{a}$, for any $a \in P^{\prime}(\Sigma)$, by $S_{a}=\Phi_{a} \sigma$. Then $\left(P^{\prime}(\Sigma), S\right)$ is an OSOL.

Proposition 17. Let $(L, S)$ be an $O S O L,(\Sigma(L), \sigma)$ be the corresponding symmetric Baer *-semigroup, and $\left(P^{\prime}(\Sigma(L)), S^{\prime}\right)$ be the OSOL of all closed projections of $\Sigma(L)$. Then the map $\varphi$ from $L$ to $P^{\prime}(\Sigma(L))$, which to each $a \in L$ assigns the Sasaki projection $\varphi_{a}$, is an OSOL isomorphism.

Proposition 18. Let

- $(\Sigma, \sigma)$ be a symmetric Baer ${ }^{*}$-semigroup;

- $\left(P^{\prime}(\Sigma), S\right)$ be the OSOL of its closed projections;

- $\left(S\left(P^{\prime}(\Sigma)\right), \sigma^{\prime}\right)$ be the symmetric Baer *-semigroup corresponding to $\left(P^{\prime}(\Sigma), S\right)$.

Then the map $\Phi$ from $(\Sigma, \sigma)$ to $\left(S\left(P^{\prime}(\Sigma)\right), \sigma^{\prime}\right)$ is a homomorphism of symmetric Baer *-semigroups.

Proposition 19. Identity (13): $g f^{\prime} g^{\prime}=g f^{\prime \prime} g^{\prime}$ holds in every symmetric Baer ${ }^{*}$-semigroup, and in every Baer ${ }^{*}$-semigroup $\Sigma(L)$ coordinatizing an $O M L L$, but fails in some Baer *-semigroups.

Proof. If $(\Sigma, \sigma)$ is a symmetric Baer ${ }^{*}$-semigroup, then by (SB2) and (SB1), we have

$$
g f^{\prime} g^{\prime}=g f^{\sigma} g^{\prime}=g f^{\prime \sigma} g^{\prime}=g f^{\prime \prime} g^{\prime} .
$$

In any Baer *-semigroup, identity (13) is equivalent to $g^{\prime \prime} f^{\prime} g^{\prime}=g^{\prime \prime} f^{\prime \prime} g^{\prime}$, because $g=g g^{\prime \prime}$ and $\left(g^{\prime \prime}\right)^{\prime}=g^{\prime}$. In a Baer ${ }^{*}$-semigroup of the form $\Sigma(L)$, this last identity is equivalent to

(14) $\varphi_{a^{\perp}} \circ \varphi_{b} \circ \varphi_{a}=\varphi_{a^{\perp}} \circ \varphi_{b^{\perp}} \circ \varphi_{a}$,

where $a, b$ are arbitrary elements of $L$. If $x \in L$ and $y=\varphi_{a}(x)$ then:

$$
\left(\left(y \vee b^{\perp}\right) \wedge b\right) \vee a=\left(\left(\left(y \vee b^{\perp}\right) \wedge b\right) \vee y\right) \vee a=\left(\left(y \vee b^{\perp}\right) \wedge(y \vee b)\right) \vee a,
$$

hence $\left(\left(y \vee b^{\perp}\right) \wedge b\right) \vee a=\left((y \vee b) \wedge b^{\perp}\right) \vee a$, from which we easily deduce identity (14). If $H$ is a Hilbert space, then the set $B(H)$ of all bounded linear operators on $H$ is a Baer *-ring, hence in particular a Baer ${ }^{*}$-semigroup. If $f$, $g \in B(H), g f^{\prime \prime} g^{\prime}=g\left(1-f^{\prime}\right) g^{\prime}=-g f^{\prime} g^{\prime}$, therefore, if $g f^{\prime} g^{\prime} \neq 0$ (which is generally true), then $g f^{\prime \prime} g^{\prime} \neq g f^{\prime} g^{\prime}$.

\section{CONCLUDING REMARKS}

(a) Proposition 19 shows that the class of all Baer *-semigroups is not the smallest equational class containing all Baer ${ }^{*}$-semigroups of the form $\Sigma(L)$.

(b) Let $H$ be a Hilbert space of dimension $\geq 2$ over $K$ (where $K=\mathbf{R}$ or C), and let $\Sigma$ be the Baer ${ }^{*}$-semigroup $B(H)$. It is well known that the canonical homomorphism $\Phi$, from $\Sigma$ to $\Sigma\left(P^{\prime}(\Sigma)\right)=\Sigma(C(H))$ is neither one-to-one, nor onto. Indeed, if $f \in B(H)$ and $X \in C(H), \Phi_{f}(X)$ is the topological closure of $f(X)$, hence for any $\lambda \in K-\{0\}, \Phi_{\lambda f}=\Phi_{f}$. On the other hand, if $A$ is an atom of $C(H)$, the mapping $\theta$ from $C(H)$ to $C(H)$, defined by $\theta(X)=\{0\}$ if $X \leq A, \theta(X)=H$ otherwise, belongs to $\Sigma(C(H))-\Phi(\Sigma)$ (if $B$ is an atom of $C(H)$, such that $B \neq A$ then $\theta(B)=H$ is not an atom). This shows that the canonical functor between OMLs and Baer ${ }^{*}$-semigroups is not an equivalence of categories. 
If for each $f \in \Sigma$ we define $f^{\sigma}$ as being the orthogonal symmetry corresponding to $\operatorname{Ker}(f)$, then $(\Sigma, \sigma)$ is not a symmetric Baer ${ }^{*}$-semigroup, but it verifies all identity axioms of these algebras except (SB1) (a) and (SB2), since $f^{\prime \sigma}=-f^{\sigma}$ and $g^{\prime \prime} f^{\sigma} g^{\prime}=2 g^{\prime \prime} f^{\prime} g^{\prime}$. If $G$ is a multiplicative subgroup of $K-\{0\}$, which is stable under conjugation (in the case where $K=\mathbf{C}$ ), we define an equivalence $R$ on $\Sigma$ by:

$$
(f R g) \Leftrightarrow(\exists \lambda \in G \text { such that } f=\lambda g)
$$

Then $R$ is a congruence on $(\Sigma, \sigma)$. Let $\left(\Sigma^{\prime}, \sigma^{\prime}\right)=(\Sigma, \sigma) / R$. It is clear that if $\{-1,2\}$ is contained in $G$, then $\left(\Sigma^{\prime}, \sigma^{\prime}\right)$ is a symmetric Baer ${ }^{*}$-semigroup, and that $P^{\prime}\left(\Sigma^{\prime}\right)=C(H)$. If $G \neq K-\{0\}$ (for instance if $G$ is the subgroup generated by $\{-1,2\})$, then we can prove in the same way as above that the homomorphism $\Phi$ from $\Sigma^{\prime}$ to $\Sigma(C(H))$ is neither one-to-one, nor onto. It follows that if we replace Baer ${ }^{*}$-semigroups by symmetric Baer ${ }^{*}$-semigroups, or by Baer *-semigroups in which identity (13) holds, then the functor defined as above is still not an equivalence of categories.

(c) Now we are going to describe a simple idealized experiment which shows that, in quantum mechanics, some orthogonal symmetries on the phase space have a physical meaning. In the classical two-hole experiment, we suppose that behind the two-hole screen, there is a second (absorbing) screen with one hole at a point of a dark interference fringe. According to whether one hole of the first screen or the other is filled up, we obtain behind the second screen, two opposite wave functions, whereas before the first screen the wave functions are identical. If we consider the distance between the two screens (which can be very small) to be negligible, we obtain two symmetrical wave functions, which separately, are physically indistinguishable; but if both holes are open, then the wave function is the superposition of the above two functions, hence is the same before the first screen and vanishes behind the second screen. If the distance between the two screens is not considered to be negligible, we obtain, by orthogonal projection onto the subspace of all functions vanishing between the two screens, an orthogonal symmetry on this closed subspace of the phase space.

\section{ACKNOWLEDGMENT}

I would like to thank Sylvia Pulamannová for several interesting suggestions.

J. Hamhalter and M. Navara have already obtained other results on OSOLs after I had given a talk in Prague on this topic. They have proved that there exist an infinite modular ortholattice admitting no OSOL structure, and that the OSOL structure on a Hilbertian lattice is unique.

\section{REFERENCES}

1. D. H. Adams, Equational classes of Foulis semigroups and orthomodular lattices, Proc. Lattice Theory Conf. (J. Schmidt, ed.), Houston, 1973, pp. 486-497.

2. E. Beltrametti and G. Cassinelli, The logic of quantum mechanics, Addison-Wesley, 1981.

3. C. C. Chang and H. J. Keisler, Model theory, North-Holland, Amsterdam, 1976.

4. D. J. Foulis, Baer ${ }^{*}$-semigroups, Proc. Amer. Math. Soc. 11 (1960), 648-654.

5. __ A note on orthomodular lattices, Portugaliae Math. 21 (1962), 65-72.

6. H. Gross and U. M. Künzi, On a class of orthomodular quadratic spaces, L'Enseign. Math. 31 (1985), 187-212. 
7. R. J. Greechie and S. P. Gudder, Quantum logics, The Logico-Algebraic Approach to Quantum Mechanics, Vol. 1 (C. A. Hooker, ed.), D. Reidel, Dordrecht-Holland, 1975, pp. 545575.

8. S. S. Holland Jr., The current interest in orthomodular lattices, The Logico-Algebraic Approach to Quantum Mechanics, Vol. 1 (C. A. Hooker, ed), D. Reidel, Dordrecht-Holland, 1975, pp. 437-496.

9. G. Kalmbach, Orthomodular lattices, Academic Press, London, 1983.

10. C. Piron, Foundation of quantum physics, Benjamin, New York, 1976.

Institut de Mathématiques et Informatique de l'ISM, Université Claude Bernard Lyon 1, 43, Bd du 11 nov. 1918, 69622 Villeurbanne Cedex, France 